EMDR-Instifut Deutschland

der autorisierte Partner des Instituts von

Francine Shapiro PhD, USA

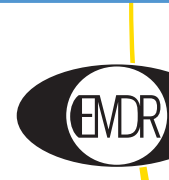

\title{
Zerhifizierte EMDR-Ausbildung
}

für Ärztl. und Psychol. Psychotherapeutlnnen und appr. KJP.

Praxisbezogene Vermittlung unter Anleitung in Kleingruppen.

Gut erreichbare Seminarorte in Deutschland,

Österreich und Schweiz.

Info und Anmeldunc:

www.emdr.de · info@emdr.de ·Tel. +49 - (0) 2204 - 25866

\section{AV Köln Akademie für Verhaltenstherapie Staatlich anerkanntes Ausbildungsinstitut}

Wir bieten

O 3- und 5-jährige Ausbildung zur / zum Psychologischen Psychotherapeutin/en

- Ausbildungsbeginn dreimal jährlich -

$O$ ärztliche Weiterbildung in Verhaltenstherapie

Fortbildungsveranstaltungen

für Approbierte Psychotherapeuten

AVT GmbH • Venloer Straße 47-53 • 50672 Köln • www.avt-koeln.org Tel.: 0221 - 2585640 • Fax: 0221 - 25856420 • info@avt-koeln.org

\section{Institut für Fort- und Weiterbildung}

in klinischer Verhaltenstherapie e. V.

Staatlich anerkanntes Ausbildungsinstitut

\section{Weiterbildungen}

$\rightarrow$ Schematherapie Kinder/Jugendliche (WS 2-4)

WS 2 am 17./18.01.2020

WS 3 am 20./21.03.2020

WS 4 am 19./20.06.2020

$\rightarrow$ Schematherapie Erwachsene (WS 1-3) vom 09.11.-13.11.2020

$\rightarrow$ Curriculum Traumatherapie (Fachkunde EMDR)

geplanter Start Oktober 2020

Fortbildungstage

$\rightarrow$ Resilienzförderung und CIPBS am 21.03.2020

Bewerbungen/Anmeldungen ab sofort möglich

Informationen und weitere Fortbildungsangebote:

IFKV - Kurbrunnenstr. 21 a - 67098 Bad Dürkheim - Tel. 06322 94828-0,

Fax: 06322 94828-29.E-mail: info@ifkv.de.Internet: www.ffkv.de

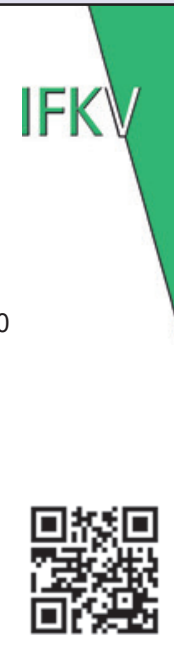




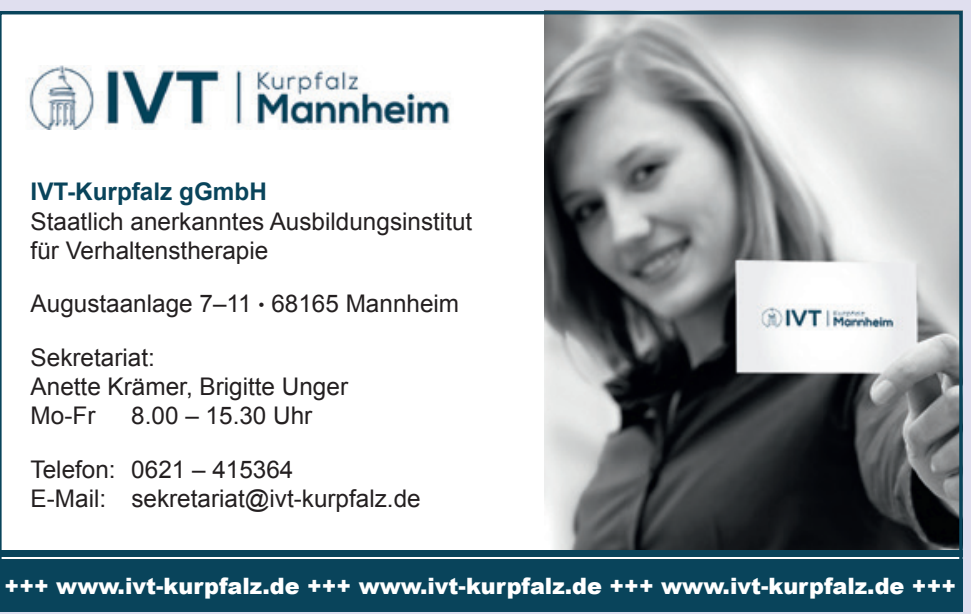

Europäische Akademie für Paartherapie (EAPT)

Einjährige Fortbildung auf Mallorca

nach humanistisch-psychodynamischen Grundsätzen

5 Blöcke á 4 Tage mit einem Übungswochenende nach Block 4 :

(176 Stunden, akkreditiert) Beginn: Sommer 2021

Leitung: Prof. Dirk Revenstorf, Halko Weiss, Ph.D.

Information: www.paartherapie-akademie.de

Anmeldung: www.meg-tuebingen.de

MH Erickson-Akademie für Klinische Hypnose (MEG)

Fortbildung in Ericksonscher Hypnose

8 Grundseminare (250 Stunden akkreditiert): Beginn: 3.-4. April 2020

Aufbaukurse zur klinischen Anwendung (akkreditiert)

Alle Seminare sind auch einzeln belegbar.

Leitung: Prof. Dirk Revenstorf und Dr. Elsbeth Freudenfeld

Information \& Anmeldung: www.meg-tuebingen.de

\section{Verhaltenstherapie}

$$
\text { Praxis | Forschung | Perspektiven }
$$

\section{Hier könnte Ihr Fort- und Weiterbildungsangebot stehen!}

Nutzen Sie Verhaltenstherapie zur effektiven

Kommunikation mit Ihrer Zielgruppe!

$$
\begin{aligned}
& \text { Informationen und Angebote bei } \\
& \text { S. KARGER Verlag GmbH } \\
& \text { Ellen Zimmermann } \\
& \text { Tel. +49 } 76145207-17 \\
& \text { e.zimmermann@karger.com }
\end{aligned}
$$




\section{FIKV}

FIKV - Fortbildungsinstitut für

Staatlich anerkanntes Ausbildungsinstitut

angegliedert an das MEDIAN Zentrum für Verhaltensmedizin Bad Pyrmont

Wir bieten:

- 3-jährige Vollzeitausbildung zum/r Psychologischen Psychotherapeuten/in

3-jährige Tätigkeit auf entsprechend vergüteten Stellen als

"Postgraduierte Psychologen» in der Klinik

- Sämtliche Ausbildungsbausteine werden in unserer Klinik bzw. in

unserem Ausbildungsinstitut durchgeführt.

- Die Kosten der Ausbildung tragen sich durch zusätzliche finanzielle

Unterstützung der Klinik und durch Einnahmen in der Ausbildungsambulanz.

- Ausbildungsbeginn jederzeit möglich

Information:

FIKV, Bombergallee 11, 31812 Bad Pyrmont

Tel. 05281 / 606763 oder 619620

sekretariat@fikv.de; www.fikv.de

\section{Ausbildung in Kinder- \\ und Jugendlichen \\ Psychotherapie mit \\ Schwerpunkt \\ Verhaltenstherapie}

\begin{tabular}{|c|}
\hline Der Kurs \\
$2020 / 2021$ \\
startet im \\
Mai 2020 \\
\hline
\end{tabular}

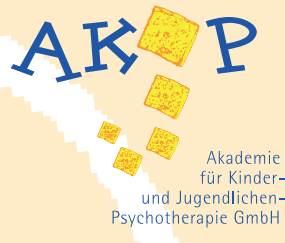

Staatlich anerkanntes Ausbildungsinstitut

[Voraussetzung: Hochschulabschluss Pädagogik/Psychologie]

- Ausbildung in Kinder- und Jugendlichenpsychotherapie

- Qualifikation in Gruppenpsychotherapie

für Kinder, Jugendliche und Familien

- Einblick und Praktikumsmöglichkeit im Bereich Neurofeedback

für ADHS-Behandlung im Rahmen der freien Spitze

- Individuelle Betreuung und Anleitung in der praktischen Ausbildung

- Große Institutsambulanz mit 16 Behandlungsräumen

- Möglichkeit im Lehrpraxenmodell zu arbeiten

27. August 2020 bis 31.08 .2020 :

Gruppentherapie-Fortbildung

Kinder-VT-Gruppe

\begin{tabular}{|c|}
\hline $\begin{array}{c}27.08 .2020 \\
\text { bis } \\
31.08 .2020\end{array}$ \\
\hline
\end{tabular}

Abrechnungsgenehmigung Kinderverhaltenstherapie

in Gruppen, 50 Theoriestunden

www.kinderverhaltenstherapie.de

Ausbildungsinstitut seit 1999 mit über 200 erfolgreich approbierten Absolventen 